Review Article

\title{
Optimum Antithrombotic Therapy in Patients Requiring Long-Term Anticoagulation and Undergoing Percutaneous Coronary Intervention
}

\author{
Nayan Agarwal, Dhruv Mahtta $(\mathbb{D}$, Cecil A. Rambarat,

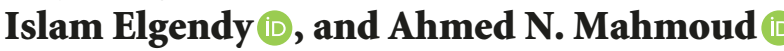 \\ Department of Medicine, University of Florida, Gainesville, FL, USA \\ Correspondence should be addressed to Ahmed N. Mahmoud; ahmed.mahmoud@medicine.ufl.edu
}

Received 30 June 2017; Accepted 14 November 2017; Published 25 March 2018

Academic Editor: Farhad Kamali

Copyright (C) 2018 Nayan Agarwal et al. This is an open access article distributed under the Creative Commons Attribution License, which permits unrestricted use, distribution, and reproduction in any medium, provided the original work is properly cited.

Management of patients on long-term anticoagulation requiring percutaneous coronary intervention is challenging. Triple therapy with oral anticoagulant and dual antiplatelet therapy is the standard of care. However, there is no strong evidence to support this strategy. There is emerging data regarding the safety and efficacy of dual therapy with oral anticoagulant and single antiplatelet therapy in these patients. In this comprehensive review we highlight available evidence regarding various antithrombotic regimens' efficacy and safety in patient with coronary artery disease undergoing percutaneous coronary intervention with long-term anticoagulation therapy requirements.

\section{Background}

Patients with mechanical heart valves, a prior systemic thromboembolic event and atrial fibrillation/flutter (AF), often require long-term anticoagulation $[1,2]$. About $20 \%$ to $30 \%$ of these patients have concomitant ischemic heart disease requiring percutaneous coronary intervention and stent implantation (PCI) [3-5]. This would mandate the use of dual antiplatelet therapy (DAPT) (aspirin and an adenosine diphosphate antagonist) for prevention of stent thrombosis and adverse events following PCI [6]. It is often a clinical dilemma, whether to use dual therapy (DT) with either oral anticoagulant (OAC) and single antiplatelet therapy (SAPT) or DAPT or triple therapy (TT) with OAC and DAPT in these patients [7].

Although primary intent of TT is to decrease the incidence of major adverse cardiac events (MACE), especially stent thrombosis, it has been found to be associated with a high annual risk of bleeding $[8,9]$, which in turn is strongly associated with recurrent hospitalization and increased morbidity and mortality $[10,11]$. Recently, new evidence has emerged questioning the benefit of TT and suggesting a regimen of dual therapy (DT) with OAC and a single antiplatelet (SAPT) agent might be equally efficacious to TT with a lower incidence of major bleeding $[8,9,12]$. There is also emerging evidence that use of DAPT in these patients is associated with similar outcomes to TT with less bleeding $[13,14]$. Hence, we attempted to review the available evidence with regard to different antithrombotic regimes in patients on long-term OAC requiring PCI.

\section{Pathophysiology of Thrombogenesis in AF and in Acute Coronary Syndrome/PCI Patients}

$\mathrm{AF}$ is the most common indication for $\mathrm{OAC}$ in patients on $\mathrm{OAC}$ requiring PCI. AF significantly increases the risk of thromboembolism $[1,15]$. The type of thrombus in $\mathrm{AF}$ is mainly fibrin rich where platelets play a smaller role $[16,17]$. Loss of atrial contraction causes stasis of blood flow in left atrium. There is also increased local expression in the dysfunctional atrial endocardium of prothrombotic molecules, such as tissue factor [18] and Von Willebrand factor (VWF) [19]. This indicates that inhibition of coagulation remains the mainstay in preventing AF related thromboembolism.

The pathogenesis of coronary thrombosis in patients with coronary artery disease (CAD) and those undergoing PCI 
is considered to be largely platelet driven. Under normal circumstances the endothelium is antithrombotic by expressing inhibitors of platelet activation, like nitric oxide (NO) and prostacyclin (PGI2), coagulation inhibitors, like tissue factor pathway inhibitor, and heparin sulphate, in addition to tissue-type plasminogen activator promoting fibrinolysis. However, when superficial erosions occur, the endothelium is activated towards hemostasis, becoming prothrombotic with expression of VWF and plasminogen activator inhibitor-1, in addition to reduced expression of NO and PGI2 [20]. This promotes platelet activation, which in turn can activate coagulation on the platelet surface. This suggests that platelet inhibition is the mainstay for avoiding plaque rupture or coronary stent induced coronary thrombosis.

\section{Antithrombotic Regimens}

3.1. Triple Therapy. Aspirin has always been cornerstone in treating ACS and/or PCI, OAC is needed for stroke prevention in $\mathrm{AF}$, mechanical heart valves, and previous thromboembolism, and a P2Y12 inhibitor is essential for prevention of stent thrombosis. Current American College of Cardiology (ACC)/American Heart Association (AHA) [6] and European Society of Cardiology [4] guidelines both recommend TT in patients with an indication for anticoagulation undergoing PCI. However, this approach may result in excess major bleeding, with rates of $2.2 \%$ within the first month and 4 to $12 \%$ within the first year of treatment [21]. These guidelines are based mostly upon observational trials and expert opinion due to the scarcity of randomized data. However, the guidelines emphasize that the treatment period should be as short as possible because of the increased bleeding risk over time. The ISAR-TRIPLE (Intracoronary Stenting and Antithrombotic Regimen-Testing of a 6-Week Versus a 6-Month Clopidogrel Treatment Regimen in Patients With Concomitant Aspirin and Oral Anticoagulant Therapy Following Drug-Eluting Stenting) [22] failed to show a benefit of 6 months of TT over 6 weeks with regard to composite of death, myocardial infarction, definite stent thrombosis, stroke, or major bleeding. An explanation for this finding may be that approximately one-half of all bleeding events occurred in the first 6 weeks after PCI, when both groups received the same therapy consisting of aspirin, clopidogrel, and OAC.

Most of the studies evaluating the role of TT in patients with an indication for OAC requiring PCI used warfarin as the OAC [9, 23-26]. Although not supported by robust clinical data, guidelines recommend a target international normalized ratio (INR) to be between 2 and 2.5 when warfarin is used $[1,6]$. Data regarding newer oral anticoagulant agents (NOACs) in patients requiring $\mathrm{OAC}$ and undergoing PCI is scant. The dose of NOACs in TT is also debatable. The ATLAS-ACS-TIMI 46 (Anti-Xa Therapy to Lower Cardiovascular Events in Addition to Standard Therapy in Subjects with Acute Coronary Syndrome-Thrombolysis in Myocardial Infarction 51) [27] demonstrated that standard $20 \mathrm{mg}$ dose of rivaroxaban when added to DAPT in ACS patients significantly increased bleeding. In ATLAS-ACS TIMI 51 trial [28] a very low dose of rivaroxaban ( $2.5 \mathrm{mg}$ twice daily) was associated with lower rates of death from cardiovascular causes, myocardial infarction, and stroke compared to DAPT alone in ACS patients without an increase in fatal bleeding, but this low dose of $2.5 \mathrm{mg}$ twice daily has not been studied for prevention of stroke in AF patients. The usage of apixaban for ACS was first explored in the Apixaban for Prevention of Acute Ischemic and Safety Events (APPRAISE) trial, which did not show a benefit with apixaban in addition to standard post-ACS treatment but resulted in significantly increased bleeding [29]. Dabigatran also increased the risk of bleeding when added to DAPT in ACS patient [30]. The ACC/AHA guidelines have not made any specific recommendations regarding NOACS. European guidelines [4] give NOACS the same level of recommendation as warfarin and suggest using lower dose (dabigatran $110 \mathrm{mg}$ twice daily, rivaroxaban $15 \mathrm{mg}$ daily, or apixaban $2.5 \mathrm{mg}$ twice daily) for patients requiring TT.

Role of rivaroxaban was investigated in PIONEERAF PCI (Open-Label, Randomized, Controlled, Multicenter Study Exploring Two Treatment Strategies of Rivaroxaban and a Dose-Adjusted Oral Vitamin K Antagonist Treatment Strategy in Subjects with Atrial Fibrillation who Undergo Percutaneous Coronary Intervention) [12] as triple therapy, which showed a rivaroxaban based strategy resulting in similar efficacy with less bleeding compared to warfarin based strategy. Most recently, efficacy of DT with dabigatran versus TT was assessed by a team of researchers from the RE-DUAL PCI (Randomized Evaluation of Dual Therapy with Dabigatran versus Triple Therapy Strategy with Warfarin in Patients with nonvalvular atrial fibrillation that have undergone PCI with Stenting) trial [31]. This was multicentered, randomizedcontrol trial consisting of 2725 patients where the primary end point was major or clinically relevant nonmajor bleeding event while the secondary endpoint consisted of thromboembolic events. At their mean follow-up period of 14 months, the authors concluded the risk of bleeding being lower among the patients in the DT with dabigatran cohort as compared to patients who received TT. DT with dabigatran was also shown to be noninferior to TT with regard to thromboembolic events. Future randomized studies such as Rivaroxaban and Ticagrelor in Atrial Fibrillation (RT-AF) [32] and Apixaban in Non-Valvular Atrial Fibrillation with a Recent Acute Coronary Syndrome or Undergoing Percutaneous Coronary Intervention (AUGUSTUS Trial) (NCT02415400) will provide more information on this subject.

There is paucity of data with regard to newer antiplatelet agents (ticagrelor and prasugrel) in combination with OAC. There are concerns that it can cause more bleeding compared to clopidogrel [50]. However, such speculations regarding the superiority or even inferiority of clopidogrel versus newer antiplatelet agents have yet to be proven by clinical data from randomized controlled trials. Nonetheless, at present both ACC/AHA and ESC guidelines recommend using clopidogrel, when TT is required.

3.2. Dual Therapy with OAC and SAPT. There are several studies that evaluated the role of DT of OAC and SAPT with TT [9, 12, 24-26, 33-42]. The WOEST [9] (what is the optimal antiplatelet and anticoagulant therapy in patients with oral anticoagulation and coronary stenting) was the first 


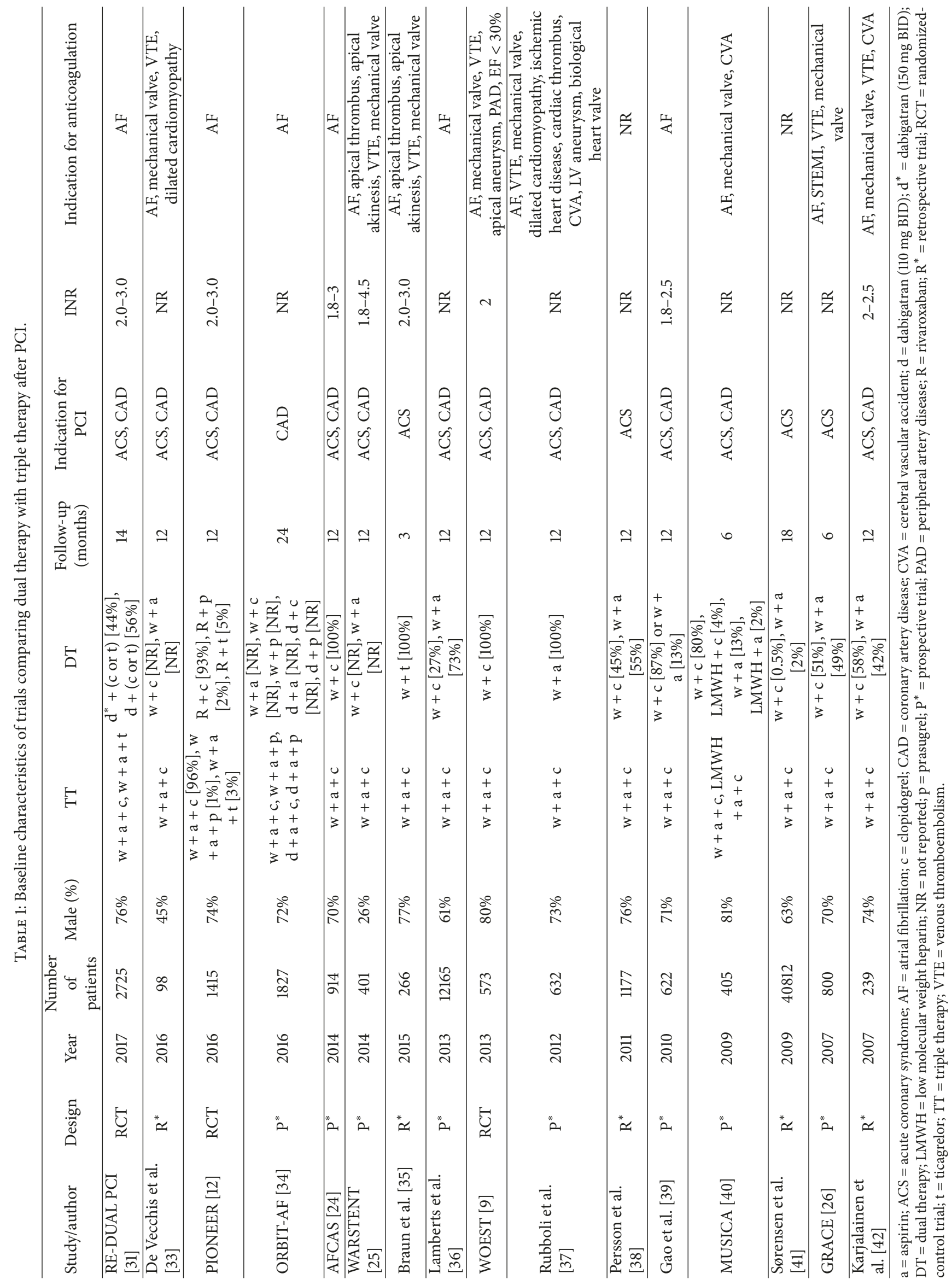


TABLE 2: Outcomes with dual therapy compared with triple therapy after PCI.

\begin{tabular}{|c|c|c|c|c|c|}
\hline Study/author & $\begin{array}{l}\text { MACE }(\%) \\
{[p \text { value }]}\end{array}$ & $\begin{array}{c}\text { Mortality }(\%) \\
\text { [p value }]\end{array}$ & $\begin{array}{l}\text { Stent thrombosis } \\
\text { (\%) }[p \text { value }]\end{array}$ & $\begin{array}{c}\text { Total bleeding }(\%) \\
\text { [ } p \text { value }]\end{array}$ & $\begin{array}{c}\text { Major bleeding }(\%) \\
\text { [ } p \text { value }]\end{array}$ \\
\hline RE-DUAL PCI [31] & NR & $\begin{array}{l}4.9 / 5.6[0.56]^{*} \\
4.6 / 3.9[0.44]^{* *}\end{array}$ & $\begin{array}{c}0.8 / 1.5[0.15] \\
0.9 / 0.9[0.98]\end{array}$ & $\begin{array}{l}42.9 / 27.1[<0.001] \\
41.4 / 33.3[<0.001]\end{array}$ & $\begin{array}{c}9.2 / 5.0[<0.001] \\
8.4 / 5.6[0.02]\end{array}$ \\
\hline $\begin{array}{l}\text { De Vecchis et al. } \\
\text { [33] }\end{array}$ & $27.1 / 12.9[0.32]$ & $8.3 / 0[0.26]$ & $2 / 0[0.59]$ & $16.7 / 19.4[0.90]$ & $8.3 / 6.5[0.89]$ \\
\hline PIONEER [12] & $6.0 / 6.5[0.75]$ & $1.9 / 2.4[0.52]$ & $0.7 / 0.8[0.79]$ & $26.7 / 16.8[<0.01]$ & $3.3 / 2.1[0.23]$ \\
\hline ORBIT-AF [34] & NR & $4.1 / 5.4[0.57]$ & NR & NR & $5.68 / 5.85[0.66]$ \\
\hline AFCAS [24] & $22 / 18[0.72]$ & $11 / 7[0.54]$ & $1 / 3[0.60]$ & $18 / 16[0.66]$ & $10 / 7[0.43]$ \\
\hline WARSTENT [25] & $16 / 15[0.98]$ & $5 / 0[0.45]$ & $1 / 0[0.76]$ & $11 / 5[0.34]$ & $4 / 5[0.84]$ \\
\hline Braun et al. [35] & NR & $3.2 / 3.8[\mathrm{NS}]$ & $0 / 0[\mathrm{NS}]$ & NR & $7 / 7.5[\mathrm{NS}]$ \\
\hline Lamberts et al. [36] & NR & 8.9/7.1 [NS] & NR & $14.3 / 10.9$ [NS] & $0.9 / 0.5[\mathrm{NS}]$ \\
\hline WOEST [9] & NR & $6.3 / 3.5[0.03]$ & $3.2 / 1.4[0.17]$ & $44.4 / 19.4[<0.01]$ & $5.6 / 3.2[0.16]$ \\
\hline Rubboli et al. [37] & $32 / 24.6[0.19]$ & $9.9 / 10.2[0.78]$ & $2.7 / 2.0[0.77]$ & NR & $5.0 / 2.6[0.32]$ \\
\hline Persson et al. [38] & NR & $3.0 / 4.2[0.43]$ & NR & $4.7 / 1.3[0.02]$ & $2.7 / 0.3[0.03]$ \\
\hline Gao et al. [39] & $8.8 / 14.9[0.01]$ & $4.4 / 5.8[0.17]$ & $0.7 / 1.7[0.73]$ & $11.8 / 7.4[0.038]$ & $2.9 / 2.5[0.73]$ \\
\hline MUSICA [40] & $23.7 / 26.1[0.001]$ & $6.8 / 10.9[0.06]$ & $4.0 / 8.7[0.04]$ & $15.5 / 13[0.02]$ & $4.3 / 6.5[0.29]$ \\
\hline Sørensen et al. [41] & NR & {$[\mathrm{NS}]$} & NR & $3.2 / 1.6[\mathrm{NS}]$ & NR \\
\hline GRACE [26] & NR & $5.1 / 6.5[0.47]$ & NR & NR & $5.9 / 4.6[0.46]$ \\
\hline $\begin{array}{l}\text { Karjalainen et al. } \\
{[42]}\end{array}$ & $21.9 / 11[0.003]$ & $8.7 / 1.8[0.003]$ & $4.1 / 1.3$ [0.09] & NR & $8.2 / 2.6[0.01]$ \\
\hline
\end{tabular}

$\mathrm{NR}$ = not reported; NS = statistically nonsignificant; number preceding "/" denotes TT (triple therapy) and number proceeding "/" denotes DT (dual therapy), TT/DT; for RE-DUAL PCI: TT/DT* ${ }^{*}$ Therapy with Dabigatran $110 \mathrm{mg}$ BID; TT/DT** = Therapy with Dabigatran $150 \mathrm{mg}$ BID.

randomized study which tested the hypothesis of using DT with OAC and SAPT in patients with an indication for longterm OAC undergoing PCI. The combination of conventional therapy of warfarin, aspirin, and clopidogrel was tested against the combination of warfarin and clopidogrel over 1 year in 573 patients. DT arm had significantly less bleeding compared to TT $(19.4 \%$ versus $44.4 \%$, HR $0.36, p<0.0001)$ and need for transfusion (3.9\% versus $9.5 \%$, OR $0.39, p=$ 0.011 ). The secondary end points of death, MI, stroke, and stent thrombosis were lower in DT compared to TT but did not reach statistical significance. In PIONEER-AF [12] the DT of low dose rivaroxaban ( $15 \mathrm{mg}$ daily) plus P2Y12 inhibitor resulted in less overall bleeding ( $16.8 \%$ versus $26.7 \%$, HR 0.59 , $p<0.001)$ and bleeding requiring medical attention $(14.6 \%$ versus $22.6 \%$, HR $0.61, p<0.001$ ) without any significant difference in the composite of cardiovascular death, MI, or stroke. The P2Y12 inhibitor was clopidogrel in $85 \%$ of cases. Danish group [36] published results of a real-life nationwide retrospective registry of 12,165 patients which showed that after 1 year, there was no increased risk of recurrent coronary events for DT (HR: $0.69 ; 95 \%$ CI: 0.48 to 1.00 ) relative to $\mathrm{TT}$, and bleeding risk was also nonsignificantly lower for warfarin plus clopidogrel (HR: $0.78,95 \%$ CI: 0.55 to 1.12 ). Moreover, there was a similar risk for all-cause mortality in patients treated with warfarin plus clopidogrel as well as those receiving triple therapy, but the combinations of warfarin plus aspirin and aspirin plus clopidogrel were associated with a significant increase in all-cause mortality compared with triple therapy. Recent meta-analyses $[8,48]$ of these observational and randomized studies have also demonstrated a similar result with less bleeding with DT without a statistically significant difference in ischemic or thromboembolic end points. Table 1 illustrates the baseline characteristics of trials comparing DAPT with TT following PCI.

As noticed in Table 1, the OAC was warfarin in majority of the studies, except for PIONEER, where rivaroxaban was used. Antiplatelet in the DT arm was clopidogrel in majority of studies, though aspirin was also used in several observational studies. Karjalainen et al. [42] demonstrated that combination of warfarin and aspirin resulted in more strokes and stent thrombosis compared to combination of warfarin and clopidogrel. Similarly in a network metaanalysis Liu et al. [46] demonstrated that the combination of OAC and clopidogrel was superior to OAC and aspirin with regard to major adverse cardiac events, stroke, MI, and all-cause mortality. Similar results were also seen in Danish registry [36] as discussed above. Newer antiplatelets (ticagrelor and prasugrel) were used only in about $15 \%$ of patients in PIONEER-AF; hence there is insufficient data regarding their use at this time. Table 2 illustrates outcomes of DAPT compared with TT following PCI.

The ESC guidelines [1] recommend an initial 4-week6-month TT based on CHADVASC and HAS-BLED score and then DT of OAC (warfarin or NOAC) and clopidogrel. WOEST [9] trial noticed that most bleeding episodes happened during the initial 180 days after PCI. Similar time frames for increased bleeding were seen in other studies of antiplatelet therapies [51, 52]. Hence, omission of the initial 4-week-6-month TT can result in reduction in bleeding as evidenced by WOEST [9] and PIONEER-AF [12]. In Table 3, we summarize the current guidelines pertaining to the use 


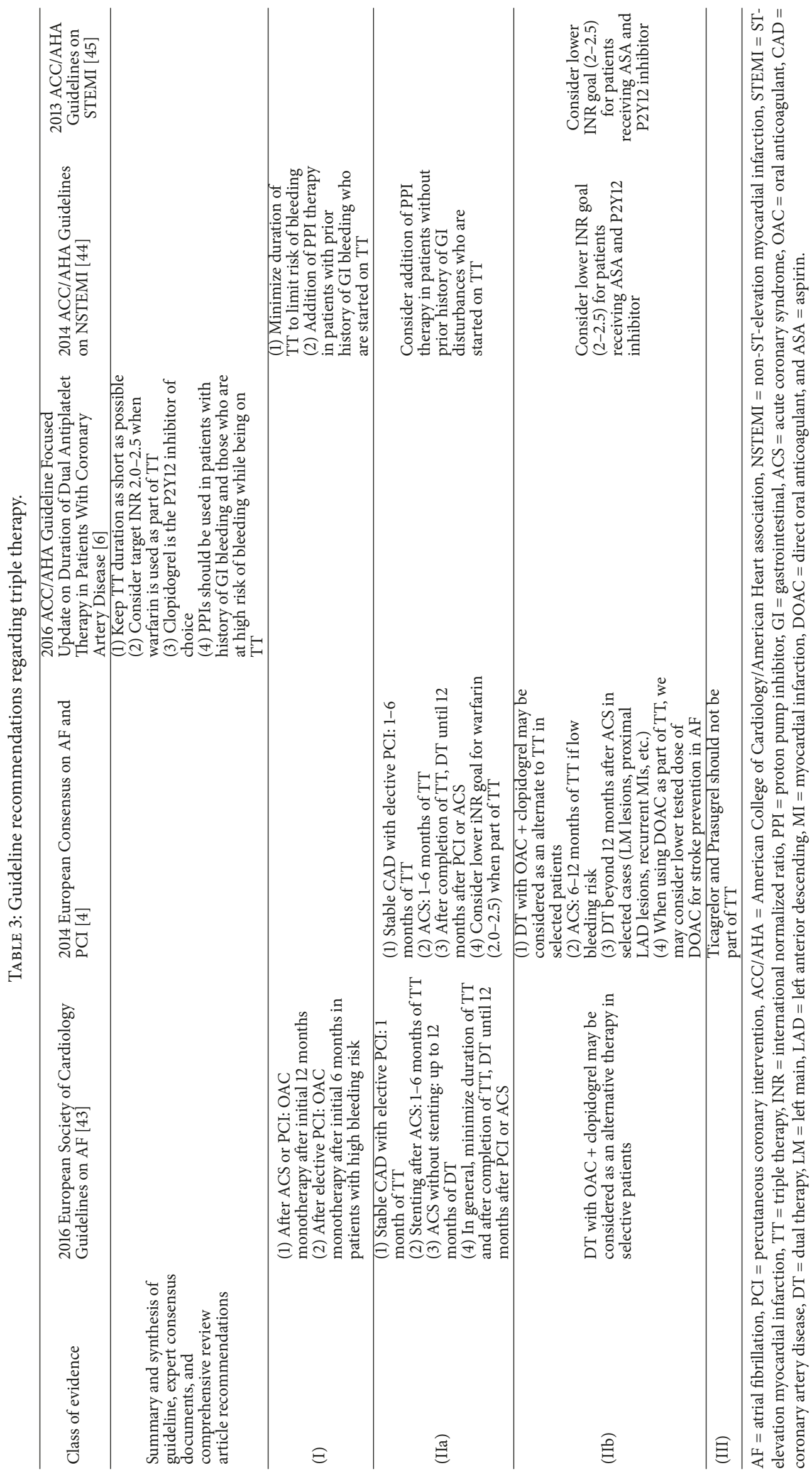




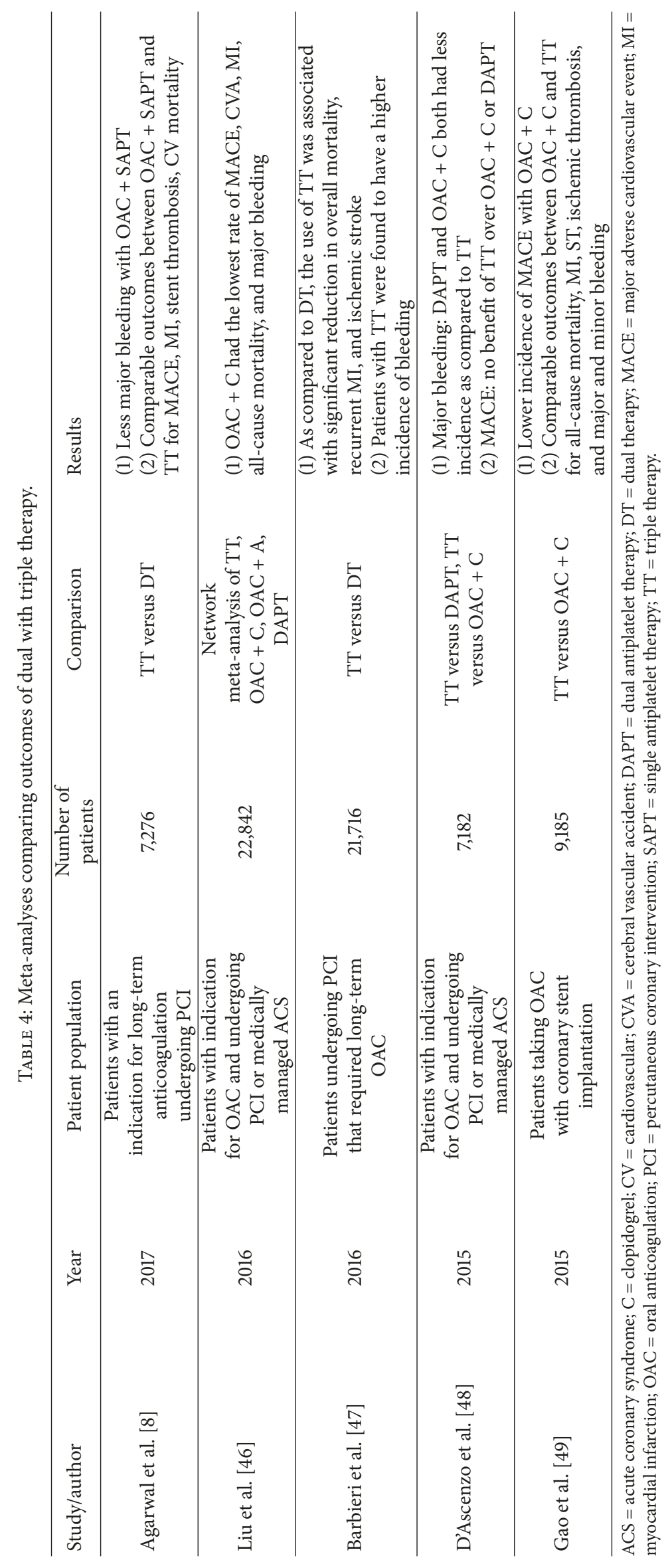


of DT and TT as recommended by ACC/AHA and ESC $[4,6,43-45]$.

3.3. Dual Therapy with DAPT. DAPT is the cornerstone of therapy in patients with ACS and/or PCI. Aspirin alone has shown to reduce the incidence of stroke by $22 \%$ in $\mathrm{AF}[53,54]$. Can DAPT provide enough benefit in stroke protection? This hypothesis was tested in ACTIVE-W study [55] (Atrial fibrillation Clopidogrel Trial with Irbesartan for prevention of Vascular Events), which showed that, compared to warfarin only, the combination of aspirin and clopidogrel resulted in a significantly increased relative risk of 1.44 (1.18-1.76; $p=0.0003$ ) of composite of stroke, systemic embolus, MI, or vascular death in patients with AF. Hence, DAPT is not considered effective for thromboembolic-prophylaxis in AF.

There are conflicting results on efficacy of DAPT in this cohort. A recent analysis published from National Cardiovascular Data Registry (NCDR) examined outcomes with DAPT versus TT in 4,959 patients $>65$ years of age with acute MI and AF who underwent PCI [14]. Relative to DAPT, patients on TT had a similar risk of major adverse cardiac events (adjusted hazard ratio [HR]: 0.99 [95\% CI: 0.86 to 1.16]), nonsignificantly lower risk of ischemic stroke (HR 0.66, 95\% CI: 0.41-1.06), but significantly greater risk of bleeding requiring hospitalization (adjusted HR: 1.61 [95\% CI: 1.31 to 1.97]), and greater risk of intracranial hemorrhage (adjusted HR: 2.04 [95\% CI: 1.25 to 3.34]). In another single center retrospective study by Choi et al. [13], it was seen that, compared to TT, DAPT had less bleeding and no difference in composite of stroke, MI, or cardiac death. Danish registry study [36], Karjalainen et al. [42], Gao et al. [39], and Rubboli et al. [37] showed a higher risk of ischemic stroke, all-cause death, and major adverse cardiac events with DAPT compared to TT and OAC plus SAPT. The NCDR analysis [14] and results of Choi et al. [13] were underpowered to detect a difference in stroke and patients had less risk factors for stroke, which could undermine the benefits of TT compared to DAPT. Network meta-analysis by Liu et al. [46] demonstrated that DAPT was associated with worse outcomes compared with TT and OAC plus clopidogrel in this patient population. Table 4 summarizes the results of various meta-analyses comparing DAPT with TT.

\section{Further Considerations for Treatment Strategies}

Based on the review of available evidence, combination of $\mathrm{OAC}$ and clopidogrel and TT are most efficacious. Combination of OAC and clopidogrel results in less bleeding compared to TT. Several large meta-analyses (Table 3 ) have suggested that OAC + SAPT either resulted in lower incidence or had equivalent rates of MACE when compared to TT. DAPT and combination of $\mathrm{OAC}$ and aspirin are inadequate with higher incidence of stroke, MI, and stent thrombosis.

Major bleeding events and blood transfusions have been associated with increased risk of death in patients undergoing PCI $[10,56]$. Majority of the patients with indication for OAC needing PCI are older $[8,56]$, who have higher thromboembolic risk, higher bleeding risk, and significant comorbidities [57]. Effect of nonmajor bleeding should not be underestimated in these patients, since even superficial or "nuisance" bleeding can lead to discontinuation of antiplatelet therapy, which may lead to subsequent thrombotic complications such as stent thrombosis $[56,58]$. Hence, a strategy to reduce bleeding is even more important in these patients.

There are several questions that still remain. Firstly, what is the optimal therapy for patients undergoing PCI who are treated with OAC and clopidogrel, once clopidogrel is discontinued? Do these patients require OAC plus aspirin or OAC alone after discontinuation of P2Y12 inhibitor? Optimal treatment strategy in this scenario is currently unknown and the lack of randomized trials assessing this troubles the daily clinician. Two large registry based studies-Danish registry [59] and ORBIT-AF (Outcomes Registry for Better Informed Treatment of Atrial Fibrillation) [60]-demonstrated that, compared to warfarin monotherapy, combination of warfarin and aspirin was associated with higher bleeding risk without reducing ischemic end points. European guidelines recommend long-term therapy with OAC only (Class $1, \mathrm{LOE} B$ ) with combination of OAC and SAPT in selected patients, for example, left main PCI, bifurcation PCI, and recurrent MI (Class 2b, LOE C). Secondly, data regarding role of NOACs in TT or DT is insufficient. PIONEER-AF showed rivaroxaban was superior to warfarin. Future trials like RTAF and AUGUSTUS will provide more data on this subject. Thirdly, the role of newer P2Y12 inhibitors like ticagrelor and prasugrel is also unclear due to lack of studies using the combination of OAC plus ticagrelor/prasugrel.

\section{Conclusions}

The efficacy of TT in patients on OAC needing PCI has never been proven. This combination increases bleeding risk, which can result in adverse patients' outcomes. New evidence, from randomized controlled trial, nationwide registries, observational studies, and meta-analysis, indicates the great potential of the combination of OAC and clopidogrel without aspirin to improve clinical outcomes in comparison with triple therapy. Therefore, OAC combined with clopidogrel seems to be a reasonable alternative to triple therapy in patients on longterm OAC who undergo PCI.

\section{Conflicts of Interest}

The authors declare that they have no conflicts of interest.

\section{References}

[1] C. T. January, L. S. Wann, J. S. Alpert et al., "2014 AHA/ACC/ HRS Guideline for the Management of Patients With Atrial Fibrillation: A Report of the American College of Cardiology/ American Heart Association Task Force on Practice Guidelines and the Heart Rhythm Society," Circulation, vol. 130, no. 23, pp. e199-e267, 2014.

[2] R. A. Nishimura, C. M. Otto, R. O. Bonow et al., "2014 AHA/ ACC Guideline for the Management of Patients With Valvular Heart Disease: Executive Summary: A Report of the American College of Cardiology/American Heart Association Task Force 
on Practice Guidelines," Circulation, vol. 129, no. 23, pp. 24402492, 2014.

[3] "A prospective survey in European Society of Cardiology member countries of atrial fibrillation management: baseline results of EURObservational Research Programme Atrial Fibrillation (EORP-AF) Pilot General Registry," Europace, vol. 16, no. 6, pp. 941-941, 2014.

[4] G. Y. H. Lip, S. Windecker, K. Huber et al., "Management of antithrombotic therapy in atrial fibrillation patients presenting with acute coronary syndrome and/or undergoing percutaneous coronary or valve interventions: A joint consensus document of the European Society of Cardiology Working Group on Thrombosis, European Heart Rhythm Association (EHRA), European Association of Percutaneous Cardiovascular Interventions (EAPCI) and European Association of Acute Cardiac Care (ACCA) endorsed by the Heart Rhythm Society (HRS) and Asia-Pacific Heart Rhythm Society (APHRS)," European Heart Journal, vol. 35, no. 45, pp. 3155-3179, 2014.

[5] S. Kralev, K. Schneider, S. Lang, T. Süselbeck, and M. Borggrefe, "Incidence and severity of coronary artery disease in patients with atrial fibrillation undergoing first-time coronary angiography," PLoS ONE, vol. 6, no. 9, Article ID e24964, 2011.

[6] G. N. Levine, E. R. Bates, J. A. Bittl et al., "ACC/AHA Guideline Focused Update on Duration of Dual Antiplatelet Therapy in Patients With Coronary Artery Disease: A Report of the American College of Cardiology/American Heart Association Task Force on Clinical Practice Guidelines: An Update of the 2011 ACCF/AHA/SCAI Guideline for Percutaneous Coronary Intervention, 2011 ACCF/AHA Guideline for Coronary Artery Bypass Graft Surgery, 2012 ACC/AHA/ACP/AATS/PCNA/ SCAI/STS Guideline for the Diagnosis and Management of Patients With Stable Ischemic Heart Disease, 2013 ACCF/AHA Guideline for the Management of ST-Elevation Myocardial Infarction, 2014 AHA/ACC Guideline for the Management of Patients With Non-ST-Elevation Acute Coronary Syndromes, and 2014 ACC/AHA Guideline on Perioperative Cardiovascular Evaluation and Management of Patients Undergoing Noncardiac Surgery," Circulation, vol. 134, pp. e123-155, 2016.

[7] G. Y. H. Lip, K. Huber, F. Andreotti et al., "Management of antithrombotic therapy in atrial fibrillation patients presenting with acute coronary syndrome and/or undergoing percutaneous coronary intervention/ stenting: A consensus document of the European Society of Cardiology Working Group on thrombosis, endorsed by the European Heart Rhythm Association [EHRA] and the European Association of Percutaneous Cardiovascular Interventions [EAPCI]," Thrombosis and Haemostasis, vol. 103, no. 1, pp. 13-28, 2010.

[8] N. Agarwal, A. Jain, A. N. Mahmoud et al., "Safety and Efficacy of Dual Versus Triple Antithrombotic Therapy in Patients Undergoing Percutaneous Coronary Intervention," American Journal of Medicine, vol. 130, no. 11, pp. 1280-1289, 2017.

[9] W. J. M. Dewilde, T. Oirbans, F. W. A. Verheugt et al., "Use of clopidogrel with or without aspirin in patients taking oral anticoagulant therapy and undergoing percutaneous coronary intervention: an open-label, randomised, controlled trial," The Lancet, vol. 381, no. 9872, pp. 1107-1115, 2013.

[10] B. J. Doyle, C. S. Rihal, D. A. Gastineau, and D. R. Holmes Jr., "Bleeding, Blood Transfusion, and Increased Mortality After Percutaneous Coronary Intervention. Implications for Contemporary Practice," Journal of the American College of Cardiology, vol. 53, no. 22, pp. 2019-2027, 2009.

[11] A. K. Chhatriwalla, A. P. Amin, K. F. Kennedy et al., "Association between bleeding events and in-hospital mortality after percutaneous coronary intervention," Journal of the American Medical Association, vol. 309, no. 10, pp. 1022-1029, 2013.

[12] C. M. Gibson, R. Mehran, C. Bode et al., "Prevention of bleeding in patients with atrial fibrillation undergoing PCI," The New England Journal of Medicine, vol. 375, no. 25, pp. 2423-2434, 2016.

[13] H. Choi, J. Ahn, S. H. Kang et al., "Prevalence, Management, and Long-Term (6-Year) Outcomes of Atrial Fibrillation Among Patients Receiving Drug-Eluting Coronary Stents," JACC: Cardiovascular Interventions, vol. 10, no. 11, pp. 1075-1085, 2017.

[14] D. Pahlajani and A. Mehta, Yearbook of Cardiology 2016, Jaypee Brothers Medical Publishers (P) Ltd., 2016.

[15] G. Y. Lip and H. S. Lim, "Atrial fibrillation and stroke prevention," The Lancet Neurology, vol. 6, no. 11, pp. 981-993, 2007.

[16] W. E. Wysokinski, S. G. Owen, D. N. Fass, D. D. Patrzalek, L. Murphy, and R. D. McBane II, "Atrial fibrillation and thrombosis: immunohistochemical differences between in situ and embolized thrombi," Journal of Thrombosis and Haemostasis, vol. 2, no. 9, pp. 1637-1644, 2004.

[17] T. Watson, E. Shantsila, and G. Y. Lip, "Mechanisms of thrombogenesis in atrial fibrillation: Virchow's triad revisited," The Lancet, vol. 373, no. 9658, pp. 155-166, 2009.

[18] Y. Nakamura, K. Nakamura, K. Fukushima-Kusano et al., "Tissue factor expression in atrial endothelia associated with nonvalvular atrial fibrillation: Possible involvement in intracardiac thrombogenesis," Thrombosis Research, vol. 111, no. 3, pp. 137-142, 2003.

[19] K. Kumagai, M. Fukuchi, J. Ohta et al., "Expression of the von Willebrand Factor in Atrial Endocardium is Increased in Atrial Fibrillation Depending on the Extent of Structural Remodeling," Circulation Journal, vol. 68, no. 4, pp. 321-327, 2004.

[20] K. W. Lee and G. Y. H. Lip, "Acute coronary syndromes: Virchow's triad revisited," Blood Coagulation \& Fibrinolysis, vol. 14, no. 7, pp. 605-625, 2003.

[21] J. S. Paikin, D. S. Wright, M. A. Crowther, S. R. Mehta, and J. W. Eikelboom, "Triple antithrombotic therapy in patients with atrial fibrillation and coronary artery stents," Circulation, vol. 121, no. 18, pp. 2067-2070, 2010.

[22] K. A. Fiedler, M. Maeng, and J. Mehilli, "Duration of triple therapy in patients requiring oral anticoagulation after drugeluting stent implantation: the ISAR-TRIPLE trial," Journal of the American College of Cardiology, vol. 65, no. 16, pp. 1619-1629, 2015.

[23] M. Lamberts, J. B. Olesen, M. H. Ruwald et al., "Bleeding after initiation of multiple antithrombotic drugs, including triple therapy, in atrial fibrillation patients following myocardial infarction and coronary intervention: a nationwide cohort study," Circulation, vol. 126, no. 10, pp. 1185-1193, 2012.

[24] A. Rubboli, A. Schlitt, T. Kiviniemi et al., "One-year outcome of patients with atrial fibrillation undergoing coronary artery Stenting: An analysis of the AFCAS registry," Clinical Cardiology, vol. 37, no. 6, pp. 357-364, 2014.

[25] A. Rubboli, F. Saia, A. Sciahbasi et al., "Outcome of patients on oral anticoagulation undergoing coronary artery stenting: Data from discharge to 12 months in the warfarin and coronary stenting (WAR-STENT) registry," The Journal of Invasive Cardiology, vol. 26, no. 11, pp. 563-569, 2014.

[26] M. C. Nguyen, Y. L. Lim, A. Walton et al., "Combining warfarin and antiplatelet therapy after coronary stenting in the Global Registry of Acute Coronary Events: Is it safe and effective to use 
just one antiplatelet agent?" European Heart Journal, vol. 28, no. 14, pp. 1717-1722, 2007.

[27] J. Mega, E. Braunwald, S. Mohanavelu et al., "Rivaroxaban versus placebo in patients with acute coronary syndromes (ATLAS ACS-TIMI 46): a randomised, double-blind, phase II trial," The Lancet, vol. 374, no. 9683, pp. 29-38, 2009.

[28] J. L. Mega, E. Braunwald, S. D. Wiviott et al., "Rivaroxaban in patients with a recent acute coronary syndrome," The New England Journal of Medicine, vol. 366, no. 1, pp. 9-19, 2012.

[29] “Apixaban after Acute Coronary Syndrome," The New England Journal of Medicine, vol. 365, no. 19, pp. 1844-1845, 2011.

[30] J. Oldgren, A. Budaj, C. B. Granger et al., "Dabigatran vs. placebo in patients with acute coronary syndromes on dual antiplatelet therapy: A randomized, double-blind, phase II trial," European Heart Journal, vol. 32, no. 22, pp. 2781-2789, 2011.

[31] C. P. Cannon, S. Gropper, D. L. Bhatt et al., "Design and Rationale of the RE-DUAL PCI Trial: A Prospective, Randomized, Phase 3b Study Comparing the Safety and Efficacy of Dual Antithrombotic Therapy With Dabigatran Etexilate Versus Warfarin Triple Therapy in Patients With Nonvalvular Atrial Fibrillation Who Have Undergone Percutaneous Coronary Intervention With Stenting," Clinical Cardiology, vol. 39, no. 10, pp. 555-564, 2016.

[32] F. Gao, H. Shen, Z. J. Wang, S. W. Yang, X. L. Liu, and Y. J. Zhou, "Rationale and design of the RT-AF study: Combination of rivaroxaban and ticagrelor in patients with atrial fibrillation and coronary artery disease undergoing percutaneous coronary intervention," Contemporary Clinical Trials, vol. 43, pp. 129-132, 2015.

[33] R. De Vecchis, C. Cantatrione, and D. Mazzei, "Clinical Relevance of Anticoagulation and Dual Antiplatelet Therapy to the Outcomes of Patients With Atrial Fibrillation and Recent Percutaneous Coronary Intervention With Stent," Journal of Clinical Medicine Research, vol. 8, no. 2, pp. 153-161, 2016.

[34] R. D. Lopes, M. Rao, D. N. Simon et al., "Triple vs Dual Antithrombotic Therapy in Patients with Atrial Fibrillation and Coronary Artery Disease," American Journal of Medicine, vol. 129, no. 6, pp. 592-599, 2016.

[35] O. Ö. Braun, B. Bico, U. Chaudhry et al., "Concomitant use of warfarin and ticagrelor as an alternative to triple antithrombotic therapy after an acute coronary syndrome," Thrombosis Research, vol. 135, no. 1, pp. 26-30, 2015.

[36] M. Lamberts, G. H. Gislason, J. B. Olesen et al., "Oral Anticoagulation and antiplatelets in atrial fibrillation patients after myocardial infarction and coronary intervention," Journal of the American College of Cardiology, vol. 62, no. 11, pp. 981-989, 2013.

[37] A. Rubboli, P. Magnavacchi, P. Guastaroba et al., "Antithrombotic management and 1-year outcome of patients on oral anticoagulation undergoing coronary stent implantation (from the registro regionale angioplastiche Emilia-Romagna registry)," American Journal of Cardiology, vol. 109, no. 10, pp. 1411-1417, 2012.

[38] J. Persson, J. Lindbäck, C. Hofman-Bang, B. Lagerqvist, U. Stenestrand, and A. Samnegard, "Efficacy and safety of clopidogrel after PCI with stenting in patients on oral anticoagulants with acute coronary syndrome," EuroIntervention, vol. 6, no. 9, pp. 1046-1052, 2011.

[39] F. Gao, Y. J. Zhou, Z. J. Wang et al., "Comparison of different antithrombotic regimens for patients with atrial fibrillation undergoing drug-eluting stent implantation," Circulation Journal, vol. 74, no. 4, pp. 701-708, 2010.
[40] A. Sambola, I. Ferreira-González, J. Angel et al., “Therapeutic strategies after coronary stenting in chronically anticoagulated patients: The MUSICA study," Heart, vol. 95, no. 18, pp. 14831488, 2009.

[41] R. Sørensen, M. L. Hansen, S. Z. Abildstrom et al., "Risk of bleeding in patients with acute myocardial infarction treated with different combinations of aspirin, clopidogrel, and vitamin $\mathrm{K}$ antagonists in Denmark: a retrospective analysis of nationwide registry data," The Lancet, vol. 374, no. 9706, pp. 1967-1974, 2009.

[42] P. P. Karjalainen, P. Porela, A. Ylitalo et al., "Safety and efficacy of combined antiplatelet-warfarin therapy after coronary stenting," European Heart Journal, vol. 28, no. 6, pp. 726-732, 2007.

[43] "Corrigendum to: 2016 ESC Guidelines for the management of atrial fibrillation developed in collaboration with EACTS", European Heart Journal, 2017.

[44] E. A. Amsterdam and N. K. Wenger, "The 2014 American College of Cardiology ACC/American Heart Association Guideline for the Management of Patients With Non-ST-Elevation Acute Coronary Syndromes," Clinical Cardiology, vol. 38, no. 2, pp. 121-123, 2015.

[45] J. L. Anderson, C. D. Adams, and E. M. Antman, "2012 ACCF/AHA focused update incorporated into the ACCF/AHA 2007 guidelines for the management of patients with unstable angina/non-ST-elevation myocardial infarction: a report of the American College of Cardiology Foundation/American Heart Association Task Force on Practice Guidelines," Circulation, vol. 127, no. 23, pp. e663-e828, 2013.

[46] J. Liu, M. Fan, J. Zhao et al., "Efficacy and safety of antithrombotic regimens after coronary intervention in patients on oral anticoagulation: Traditional and Bayesian meta-analysis of clinical trials," International Journal of Cardiology, vol. 205, pp. 89-96, 2016.

[47] L. Barbieri, M. Verdoia, A. Schaffer, H. Suryapranata, and G. De Luca, "Risk and Benefits of Triple Therapy in Patients Undergoing Coronary Stent Implantation Requiring Oral Anticoagulation: A Meta-Analysis of 16 Studies," Cardiovascular Drugs and Therapy, vol. 30, no. 6, pp. 611-622, 2016.

[48] F. D’Ascenzo, S. Taha, C. Moretti et al., "Meta-analysis of randomized controlled trials and adjusted observational results of use of clopidogrel, aspirin, and oral anticoagulants in patients undergoing percutaneous coronary intervention," American Journal of Cardiology, vol. 115, no. 9, pp. 1185-1193, 2015.

[49] X.-F. Gao, Y. Chen, Z.-G. Fan et al., "Antithrombotic Regimens for Patients Taking Oral Anticoagulation after Coronary Intervention: A Meta-analysis of 16 Clinical Trials and 9185 Patients," Clinical Cardiology, vol. 38, no. 8, pp. 499-509, 2015.

[50] N. Sarafoff, A. Martischnig, J. Wealer et al., "Triple therapy with aspirin, prasugrel, and vitamin $\mathrm{K}$ antagonists in patients with drug-eluting stent implantation and an indication for oral anticoagulation," Journal of the American College of Cardiology, vol. 61, no. 20, pp. 2060-2066, 2013.

[51] P. B. Berger, D. L. Bhatt, V. Fuster et al., "Bleeding complications with dual antiplatelet therapy among patients with stable vascular disease or risk factors for vascular disease: Results from the clopidogrel for high atherothrombotic risk and ischemic stabilization, management, and avoidance (CHARISMA) trial," Circulation, vol. 121, no. 23, pp. 2575-2583, 2010.

[52] C. Sosnowski, "Commentary to the article: Wiviott SD, Braunwald E, McCabe $\mathrm{CH}$ et al. Prasugrel versus clopidogrel in patients with acute coronary syndromem. N Engl J Med 2007; 
357: 2001-15," Kardiologia Polska, vol. 66, no. 2, pp. 222-225, 2008.

[53] "Risk Factors for Stroke and Efficacy of Antithrombotic Therapy in Atrial Fibrillation," JAMA Internal Medicine, vol. 154, no. 13, p. 1449, 1994.

[54] R. G. Hart, O. Benavente, R. McBride, and L. A. Pearce, "Antithrombotic therapy to prevent stroke in patients with atrial fibrillation: a meta-analysis," Annals of Internal Medicine, vol. 131, no. 7, pp. 492-501, 1999.

[55] S. Connolly, J. Pogue, and R. Hart, "Clopidogrel plus aspirin versus oral anticoagulation for atrial fibrillation in the Atrial fibrillation Clopidogrel Trial with Irbesartan for prevention of Vascular Events (ACTIVE W): a randomised controlled trial," The Lancet, vol. 367, no. 9526, pp. 1903-1912, 2006.

[56] W. J. M. Dewilde, P. W. A. Janssen, F. W. A. Verheugt et al., "Triple therapy for atrial fibrillation and percutaneous coronary intervention: A contemporary review," Journal of the American College of Cardiology, vol. 64, no. 12, pp. 1270-1280, 2014.

[57] T. Gomes, M. M. Mamdani, A. M. Holbrook, J. M. Paterson, C. Hellings, and D. N. Juurlink, "Rates of hemorrhage during warfarin therapy for atrial fibrillation," Canadian Medical Association Journal, vol. 185, no. 2, pp. E121-E127, 2013.

[58] P. Roy, L. Bonello, R. Torguson et al., "Impact of "Nuisance" Bleeding on Clopidogrel Compliance in Patients Undergoing Intracoronary Drug-Eluting Stent Implantation," American Journal of Cardiology, vol. 102, no. 12, pp. 1614-1617, 2008.

[59] M. Lamberts, G. H. Gislason, G. Y. H. Lip et al., "Antiplatelet therapy for stable coronary artery disease in atrial fibrillation patients taking an oral anticoagulant: A nationwide cohort study," Circulation, vol. 129, no. 15, pp. 1577-1585, 2014.

[60] B. A. Steinberg, S. Kim, and J. P. Piccini, "Use and associated risks of concomitant aspirin therapy with oral anticoagulation in patients with atrial fibrillation: insights from the Outcomes Registry for Better Informed Treatment of Atrial Fibrillation," Circulation, vol. 128, no. 7, pp. 721-728, 2013. 


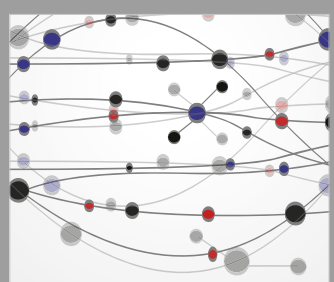

The Scientific World Journal
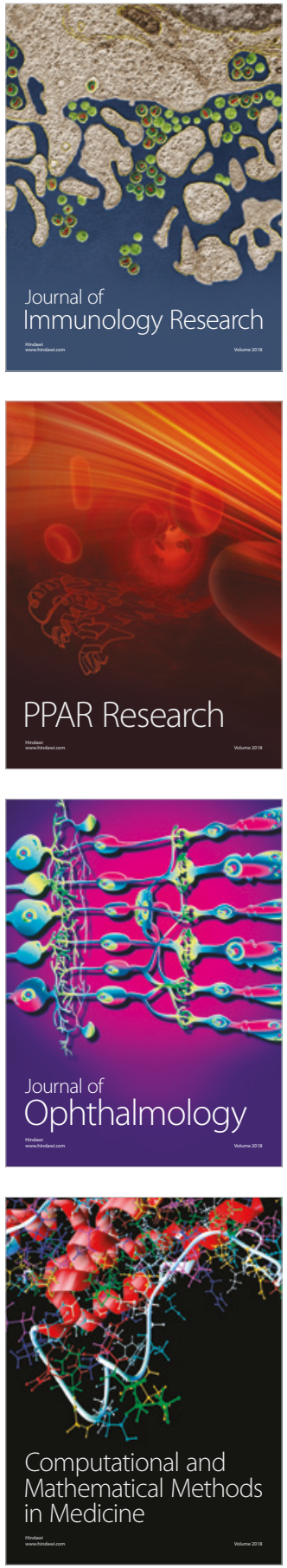

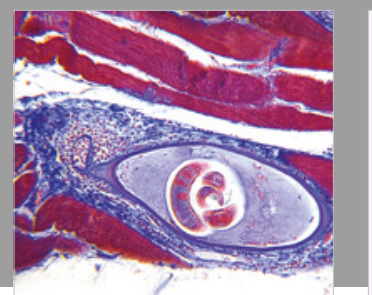

Gastroenterology Research and Practice

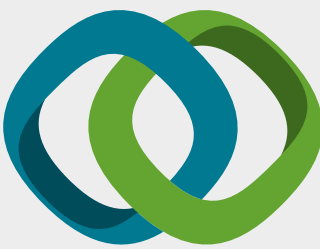

\section{Hindawi}

Submit your manuscripts at

www.hindawi.com
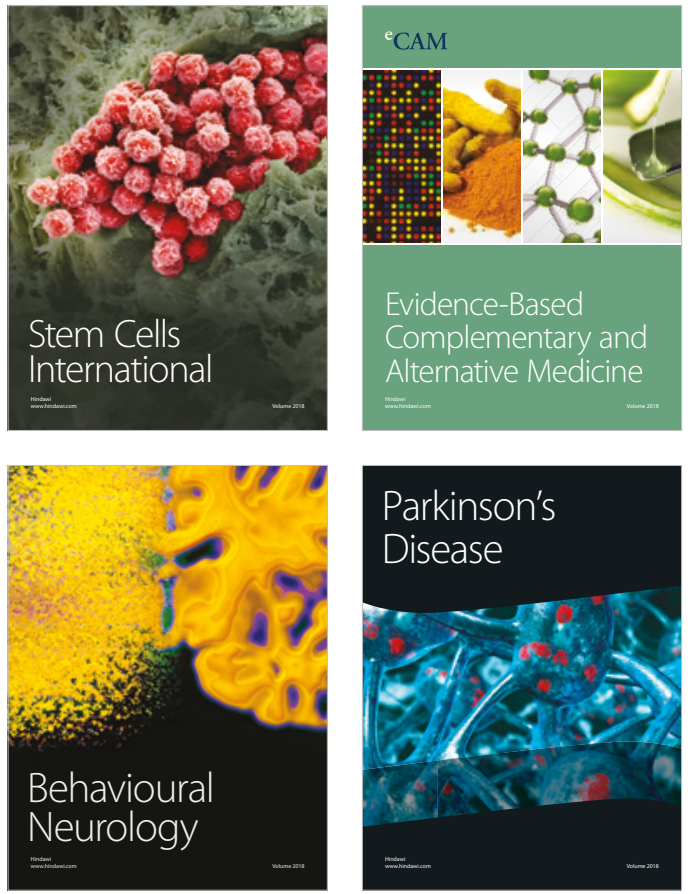

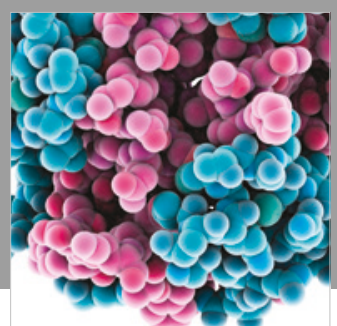

ournal of

Diabetes Research

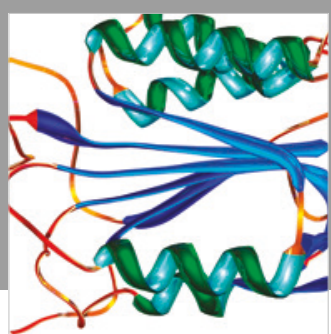

Disease Markers
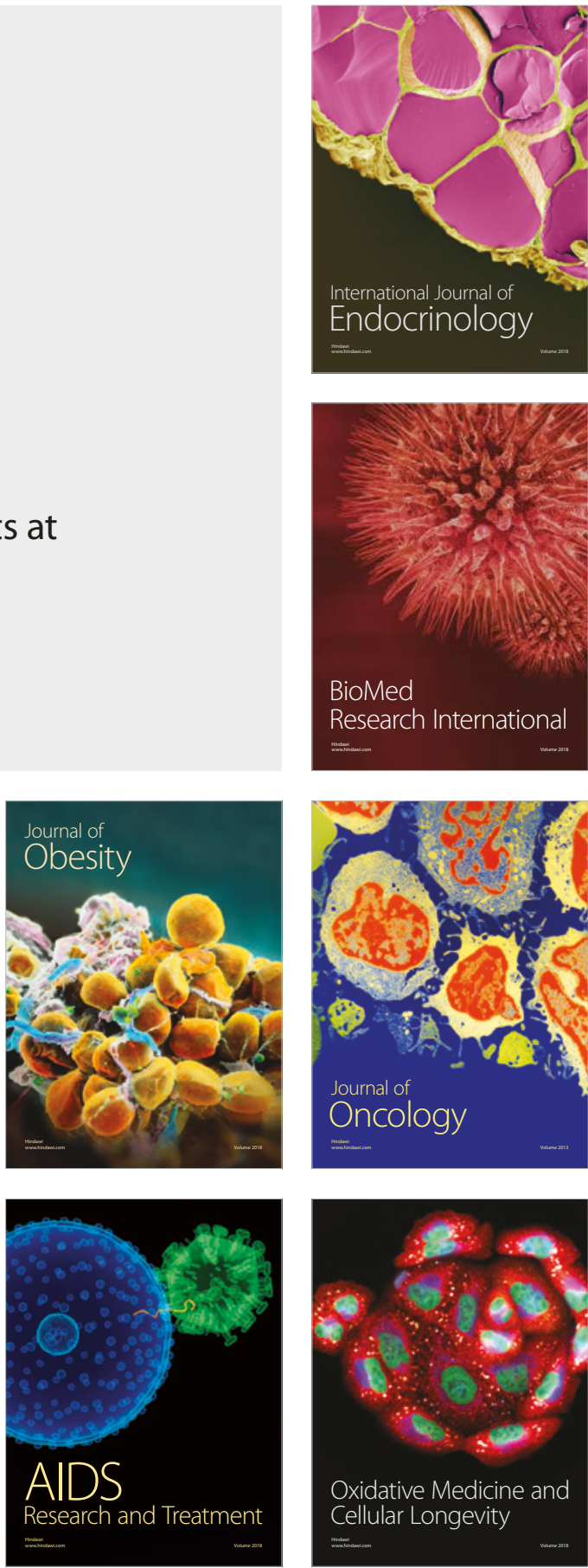